\title{
Thalamo-olivary degeneration in a patient with laryngopharyngeal dystonia
}

\author{
Toru Yamamoto, Mariko Yamashita
}

\begin{abstract}
A 67 year old woman with a two year history of laryngopharyngeal dystonia, spasmodic dysphonia, and parkinsonism succumbed to Wernicke's encephalopathy and died six months later. Necropsy showed, besides Wernicke's encephalopathy, degenerative changes in selected thalamic nuclei (dorsomedial, pulvinar, and the medial geniculate bodies) and the inferior olives and numerous cerebellar torpedoes. The substantia nigra and basal ganglia were spared. Immunostaining for prion protein was negative. This patient indicated a new type of presentation of so-called pure thalamic degeneration, or more precisely thalamo-olivary degeneration.
\end{abstract}

$(\Im$ Neurol Neurosurg Psychiatry 1995:59;438-441)

Keywords: thalamo-olivary degeneration; thalamic degeneration; spasmodic dysphonia; laryngopharyngeal dystonia

Thalamic degeneration is a rare condition, the clinical manifestations of which have been variable cognitive memory and behavioural disorders. ${ }^{1}$ Three types of selective thalamic degeneration have been distinguished ${ }^{12}$ : firstly, combination with a variety of neurodegenerative $^{3-5}$ and metabolic disorders such as Menke's kinky hair disease; secondly, the thalamic form of Creutzfeldt-Jakob disease ${ }^{2}$; thirdly, isolated or pure thalamic degeneration. ${ }^{26-15}$ Now that a mutation at codon 178 of the prion protein gene has been detected in fatal familial insomnia ${ }^{1415}$ that used to belong to the third category, ${ }^{213}$ these patients may well be recategorised to the second or prion disease group despite different clinical manifestations. Therefore the patients with pure thalamic degeneration who were negative or not tested for prion protein were only seven $^{26-11}$; among these were four Japanese and one Chinese; and two of the seven patients were familial. The thalamic involvement has been roughly selective among its divisions and there has been no patient in whom the thalamus was the only site of degeneration. Interestingly, degeneration of the inferior olives accompanied the thalamic changes not only in all the patients with pure thalamic degeneration except one ${ }^{7}$ but also in patients with prion disease. The olivary degeneration is associated with $\mathrm{no}^{267}$ or mild ${ }^{8-11}$ cerebellar cortical changes; thus a trans-synaptic degeneration was unlikely. Therefore we think that the term thalamo-olivary degeneration is more appropriate to designate this condition.

We experienced such a patient with thalamo-olivary degeneration, who presented mainly with parkinsonism, dystonia, and spasmodic dysphonia. ${ }^{16}{ }^{17}$ Our patient was the first in whom spasmodic dysphonia was one of the main features and the thalamic and inferior olivary lesions were confirmed at postmortem examination.

\section{Case report}

A 67 year old independent woman ex-typist who had had a partial gastrectomy for gastric cancer at the age of 65 gradually developed gait disturbance and involuntary laryngopharyngeal movements with a component of spasmodic dysphonia over 18 months. Her family history was not relevant. She had been living alone and her relatives had noticed subtle abnormalities in her behaviour after the surgery. For example, she refused gifts of food based on her assumption that it was old and she removed the petals from arranged flowers to detect presumed insects. Because of increasing anorexia and discomfort when breathing the patient was again admitted to our hospital in December 1992. There was no evidence of recurrence of cancer or presence of other disorders to account for her loss of appetite and a neurological consultation was made. Her mental state was unremarkable except for depression; and she scored $28 / 30$ on the mini mental state examination. Cranial nerves were intact and swallowing was not disturbed. There was moderate parkinsonism with hypokinesia, cogwheel rigidity of the limbs, neck, and trunk, a mask-like face, and a shuffling gait. Tremor was absent and postural response was intact. Tendon reflexes were normal and the plantar response was flexor. There were involuntary movements in the throat muscles that were characterised by irregular abrupt stops in the inhalation phase, 
producing faint unintentional phonation. These abnormalities were confirmed by pneumotachogram (fig 1). Fibre laryngoscopy also showed irregular, repetitive, vocal fold closing during the resting state of respiration. Synchronous with the unintentional phonation was raising of her throat as if to swallow, nodding-like neck flexion, and slight forward bending of the trunk. Ataxia was not evident. Brain CT was normal. Levodopa up to 400 $\mathrm{mg} /$ day with a decarboxylase inhibitor and an antidepressant were tried without an appreciable effect.

Fluid infusion without supplementation of vitamins induced Wernicke's encephalopathy in April 1993. Thereafter, no more spasmodic dysphonia, dystonia, or dyskinesia was observed. Although the patient partially recovered, she was kept confined to bed and her mental state deteriorated again five months after the attack of Wernicke's encephalopathy. Eventually she entered a state of akinetic

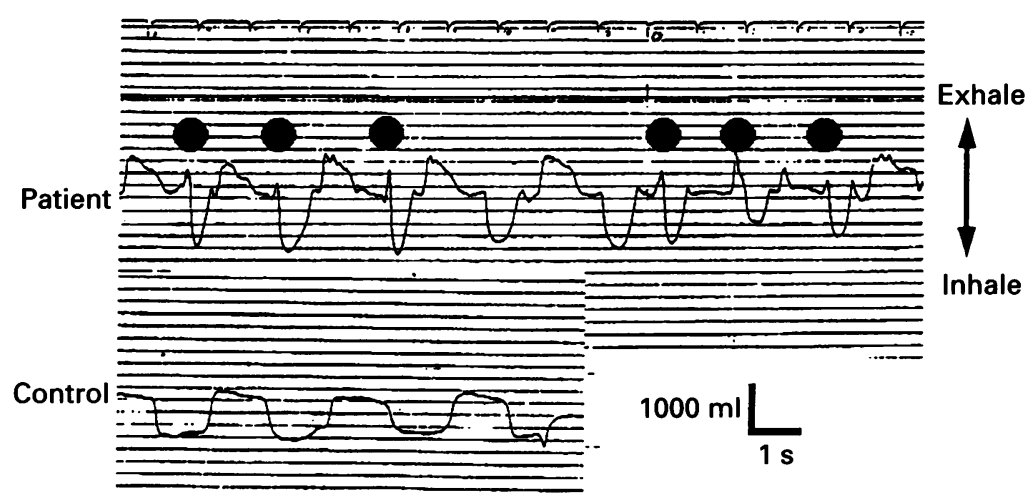

Figure 1 Pneumotachogram showing abrupt involuntary interruption (dots) of inhalation during normal respiration. At these points the patient produced faint unintentional phonation. $A$ mask was worn by the patient and normal control and air flow was measured with the same calibration.

Figure 2 Severe neuron loss and isomorphic gliosis in the dorsomedial body of the thalamus; haematoxylin-eosin. Bar represents $100 \mu \mathrm{m}$. mutism. Her ECGs showed background disorganisation with diffuse theta and delta slowing: no periodic sharp discharges ${ }^{18}$ were recorded on any occasion. She died two months later of uncontrollable respiratory infection in October 1993.

Postmortem examination showed bronchopneumonia and widespread microabscess formation. There had been no recurrence of cancer. The brain weighed $1140 \mathrm{~g}$. Macroscopic abnormalities were limited to the necrotising lesions around the third ventricle and the aqueduct and a mild atrophy of the mammillary bodies. Histological examination confirmed tissue destruction with macrophages and hypertrophic astrocytosis, which was consistent with Wernicke's encephalopathy. There was severe thalamic degeneration distinct from the changes of Wernicke's encephalopathy, consisting of isomorphic fibrillary gliosis and loss of neurons (fig 2). The dorsomedial, pulvinar, and the medial geniculate bodies were mainly affected bilaterally (fig 3). The anterior nuclei were mostly preserved. The superior and inferior colliculi were moderately gliotic. The inferior olives and the accessory olivary nuclei showed severe degeneration (fig 4). Although the cerebellar cortex escaped neuron loss, there were profuse torpedoes (fig 5). A few Lewy bodies were seen in the pigmented brainstem neurons. Otherwise, the basal ganglia, substantia nigra, subthalamus, the rest of the brainstem, and the spinal cord were intact except for scattered microabscesses and mild gliosis. A few Alzheimer's neurofibrillary tangles were seen in the hippocampus and senile plaques or ballooned neurons were not detected. Spongy changes characteristic of Creutzfeldt-Jakob disease were not found and immunostaining for prion protein was negative.

\section{Discussion}

"Pure" thalamic degeneration, even in its broad sense, has been reported in only a few cases. $^{2}$ 6-1114 The precise mapping of subdivisional thalamic involvement in these patients is hampered by variable nomenclature. According to the standard text of Carpenter and Sutin ${ }^{19}$ and the schematic illustrations in the medical literature, the most consistent affected regions were dorsomedial, anterior, ventral anterior, and pulvinar. Our patient was unique both in the affected medial geniculate body, that was intact in all except one patient reported by Hori et $a l,{ }^{10}$ and the spared anterior nuclei, that were always affected in other patients described. The constant involvement of the inferior olives in pure thalamic degeneration is a peculiar finding as there is no direct functional connection between these two structures. Of related interest both in pure thalamic degeneration and in prion disease were minor cerebellar changes that included torpedoes of the Purkinje cell axons, ${ }^{311-1320}$ which are not specific, ${ }^{21}$ but are intriguing accompaniments.

There were patients with multiple system degeneration in which thalamic changes were 
Figure 3 Schema of thalamic degeneration (black) and Wernicke's encephalopathy (stippled). Serial coronal sections from the right caudal (top left), through the rostral, to the left caudal (bottom right) portions.

$A=$ anterior

$A=$ anterior
$C a=$ caudate

$C M=$ centromedian;

$D M=$ dorsomedial

$G P=$ globus pallidus;

IC = internal capsule;

$L D=$ lateral dorsal;

$L G B=$ lateral geniculate

body;

$L P=$ lateral posterior;

Mam = mammillary body;

Midl = midline;

$M G B=$ medial geniculate

body;

$O T=$ optic tract;

$P F=$ parafascicular;

Pulv = pulvinar;

Put = putamen;

Ret $=$ reticular;

$R N=$ red nucleus;

$S C=$ superior colliculus;

SN = substantia nigra;

Sth = subthalamus;

$V A=$ ventral anterior;

$V L=$ ventral lateral;

$V P=$ ventral posterior.
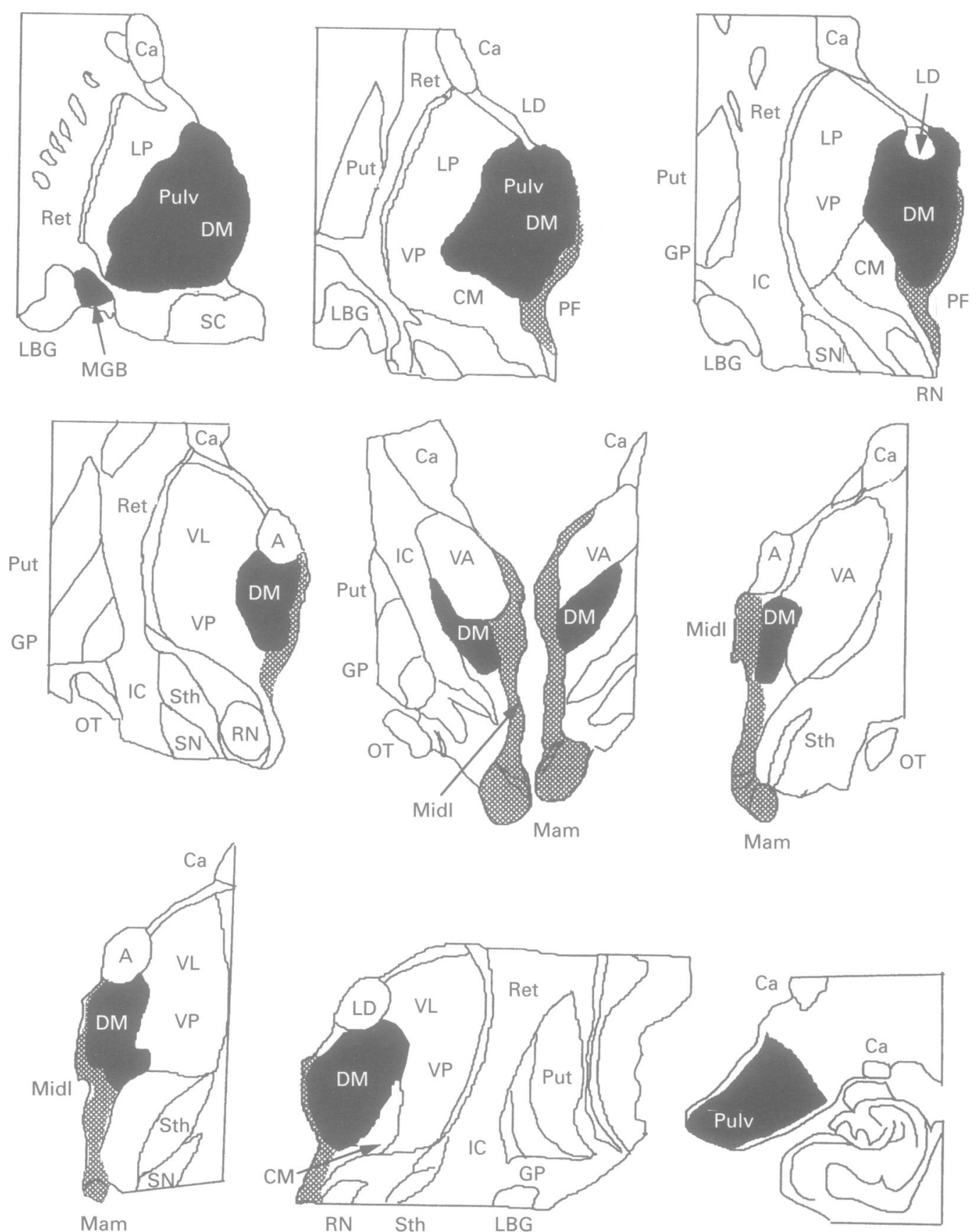

disproportionally severe. These patients ${ }^{3422}$ were not different in the distribution pattern of thalamic lesion from patients with pure degeneration. Similarly, cases of the thalamic form of prion protein disease showed changes indistinguishable from pure cases. A normal prion protein gene but positive prion protein immunoblot were seen in one patient with mild spongiform encephalopathy. ${ }^{12}$ Wernicke's encephalopathy is another disorder in which the thalami, particularly the dorsomedial and pulvinar areas, are always involved..$^{23}$ Involvement of the anterior nucleus is frequent, whereas that of the medial geniculate body is uncommon in Wernicke's encephalopathy: both of these are contrary to the findings in our patient. Furthermore, olivary degeneration is not a part of Wernicke's encephalopathy.

Clinical presentation of this patient was distinct from the other reported cases and indicates a wide clinical range of thalamic degeneration despite rather uniform pathological findings. Spasmodic dysphonia is a term that describes a family of strained, strangled voices, voice tremor, or stridor, resulting from vocal fold spasm. ${ }^{16}{ }^{17}$ Three types-adductor, abductor, and mixed-of spasmodic dysphonia are known, among which the adductor type is by far the most common. For dystonia in general, there has been uncertainty as to the underlying pathology. Not infrequently psychogenic dysfunction (psychoneurosis) is the only explanation for spasmodic dysphonia. ${ }^{24}$ Pool et al, ${ }^{16}$ however, found neurological abnormalities in about $70 \%$ of their patients. Disturbed rapid alternating movements, weakness, and tremor were some of the abnormalities. The authors suggested, from anatomical and functional brain imaging studies, that the pallidothalamic area (pars oralis of the ventro- 


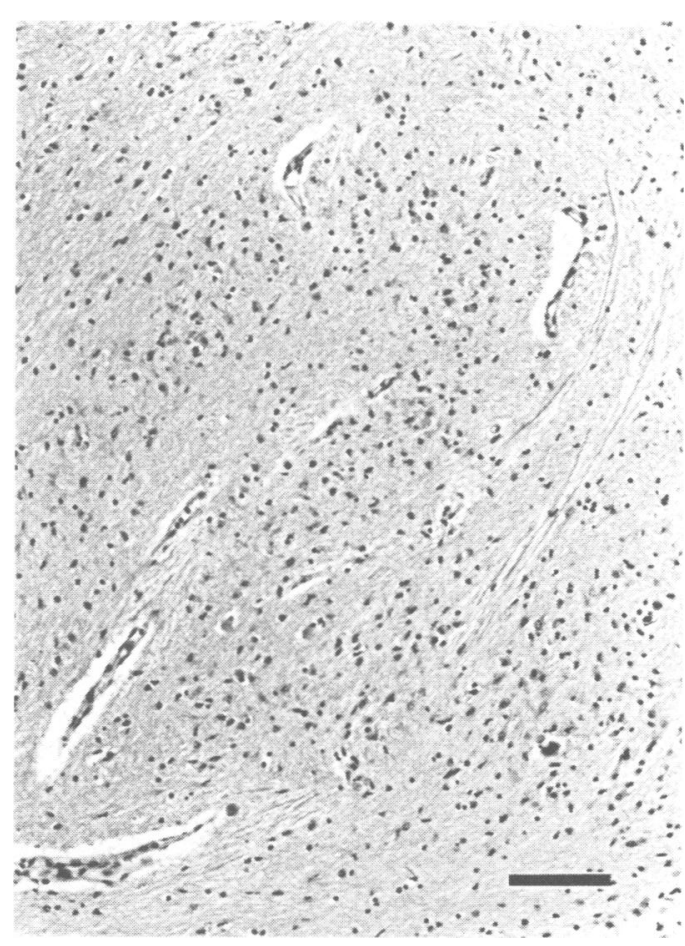

Figure 4 Severe neuronal loss and gliosis of the inferior olive; haematoxylin-eosin. Bar represents $100 \mu \mathrm{m}$.

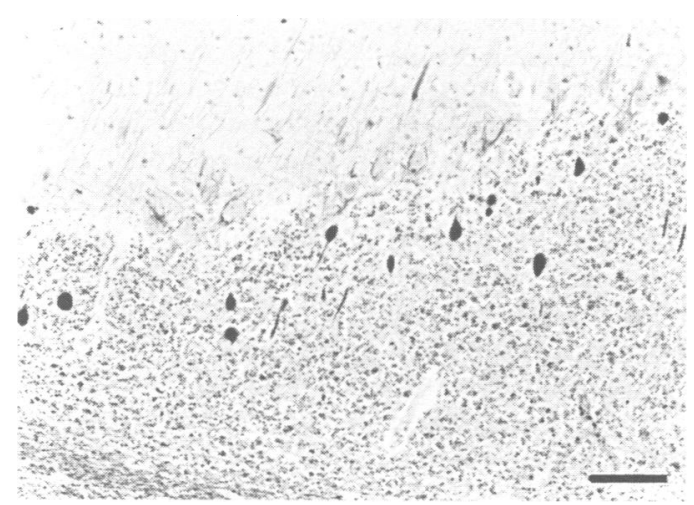

Figure 5 Numerous cerebellar torpedoes; modified Bielschowsky stain. Bar represents $100 \mu \mathrm{m}$.

lateral nucleus of the thalamus) - the supplementary motor area-could account for the neurological findings. Rosenfield et $a l^{17}$ evaluated 100 patients with spasmodic dysphonia and showed that 71 had essential tremor, 25 had Meige's syndrome, and 12 were hypothyroid. Among the reported patients with thalamic degeneration only one ${ }^{8}$ had abnormal phonation: she showed strong inspiratory effort due to whoop-like laryngeal spasms. Even this patient's sign was unlike the rhythmic spasmodic dysphonia of our patient, who showed nodding-like neck movement and pharyngeal components.

Regarding other clinical signs in thalamic degeneration, particularly different in our patient was an apparent absence of both dementia at the early stages and sleep disturbance. Superimposition of Wernicke's encephalopathy could have terminated the natural course of the disease, muting these important signs and making the laryngopharyngeal dystonia disappear. The necrotising changes in Wernicke's encephalopathy could possibly interrupt neural circuits underlying the movement disorder as in surgical procedures for tremor.

We thank Dr Jun Tateishi of Kyushu University for the prion protein study and Yuri Fujiwara, speech therapist of our hospital, for evaluating speech dysfunction.

1 Jellinger KA. Rare neurodegenerative disorders. In: Calne DB, ed. Neurodegenerative diseases. Philadelphia: WB Saunders Company, 1994:909-31.

2 Martin J. Thalamic degenerations. In: Vinken PJ, Bruyn GW, Klawans HL, de Jong JMBV, eds. Handbook of Clinical Neurology, vol 60. Amsterdam: Elsevier Science Publishers, 1991:701-15.

3 Katz DA, Naseem A, Horoupian DS, Rothner AD, Davies P. Familial multisystem atrophy with possible thalamic dementia. Neurology 1984;34:1213-7.

4 Yagishita $T$, Kojima S, Arai K, Hirayama K, Akai J, Takemura K. Dementia and disturbance of consciousness in thalamic degeneration. Brain Nerve (Tokyo) 1987; ness in tha

5 Yamamoto $\mathrm{T}$, Kawamura J, Hashimoto $\mathrm{S}$, et al. Pallidonigro-luysian atrophy, progressive supranuclear palsy and adult onset Hallervorden-Spatz disease: a case of akinesia as a predominant feature of parkinsonism. $\mathcal{F}$ Neurol $\mathrm{Sci}$ 1990;101:98-106.

6 Stern K. Severe dementia associated with bilateral symmetrical degeneration of the thalamus. Brain 1939;62: 157-71.

7 Schulman S. Bilateral symmetrical degeneration of the thalamus. A clinico-pathological study. F Neuropathol Exp Neurol 1957;16:446-70.

8 Oda M, Yoshida T, Shiraki H, Yokoyama T. An autopsy case of the systemic degeneration of the bilateral thalamic nuclei associated with olivocerebellar atrophy. Psychiatric Neurology (Tokyo) 1965;67:67-82.

9 Oda $M$. Thalamus degeneration in Japan. A review from clinical and pathological viewpoints. Appl Neurophysiol 1976/77;39:178-98.

10 Hori A, Ikeda K, Kosaka K, Shinohara S, Iizuka R. System degeneration of the thalamus. A clinico-neuropathological study. Arch Psychiatr Nervenkr 1981;231:71-80.

11 Hirano Y, Katayama S, Yokoyama S, Honma K, Nakajima S. An autopsy case of thalamic degeneration: review of the literature. Clinical Neurology (Tokyo) 1984;24: 1039-49.

12 Mizusawa H, Ohkoshi N, Sasaki H, Kanazawa I, Nakanishi $T$. Degeneration of the thalamus and inferior olives associated with spongiform encephalopathy of the cerebral ciated with spongiform encephalopa
cortex. Clin Neuropathol 1988;7:81-6.

13 Little BW, Brown PW, Rodgers-Johnson P, Perl DP, Gajdusek DC. Familial myoclonic dementia masquerading as Creutzfeldt-Jakob disease. Ann Neurol 1986;20: ing as

14 Lugaresi E, Medori $R$, Montagna $P$, et al. Fatal familial insomnia and dysautonomia with selective degeneration of thalamic nuclei. $N$ Engl $₹$ Med 1986;315:997-1003.

15 Petersen RB, Tabaton M, Berg L, et al. Analysis of the prion protein gene in thalamic dementia. Neurology 1992;42:1859-63.

16 Pool KD, Freeman FJ, Finitzo T, et al. Heterogeneity in spasmodic dysphonia. Neurologic and voice findings. Arch Neurol 1991;48:305-9.

17 Rosenfield DB, Donovan DT, Sulek M, Viswanath NS, Inbody GP, Nudelman HB. Neurologic aspects of spasmodic dysphonia. $\mathcal{F}$ Otolaryngol 1990;19:231-6.

18 Yamamoto T, Imai T. A case of diffuse Lewy body and Alzheimer's disease with periodic synchronous disAlzheimer's disease with periodic synchronous
charges. $₹$ Neuropathol Exp Neurol 1988;47:536-48.

19 Carpenter MB, Sutin J. Human neuroanatomy. 8th ed.

20 Manetto V, Medori R, Cortelli P, et al. Fatal familial insomnia: clinical and pathologic study of five new cases. Neurology 1992;42:312-9.

21 Takahashi N, Iwatsubo T, Nakano I, Machinami R. Focal appearance of cerebellar torpedoes associated with discrete lesions in the cerebellar white matter. Acto Neuropathol (Berl) 1992;84:153-6.

22 Deymeer F, Smith TW, DeGirolami U, Drachman DA. Thalamic dementia and motor neuron disease. Neurology 1989;39:58-61.

23 Victor M, Adams RD, Collins GH. The WernickeKorsakoff syndrome and related neurologic disorders due to alcoholism and malnutrition. 2nd ed. Philadelphia: FA Davis Company, 1989:61-112.

24 Stemple JC. Management of spasmodic dysphonia. In: Voice therapy: clinical studies. St. Louis: Mosby-Year Book, 1993:125-54. 